\title{
LINEAR CAMERA AUTOCALIBRATION WITH VARYING PARAMETERS
}

\author{
Antonio Valdés
}

\author{
Dpto. de Geometría y Topología \\ Universidad Complutense de Madrid \\ Ciudad Universitaria, $\mathrm{s} / \mathrm{n}$ \\ E-28040 Madrid, Spain
}

\begin{abstract}
We provide a new technique for the Euclidean upgrading of a projective calibration for a set of ten or more cameras with known skew angle and aspect ratio and arbitrary varying focal length and principal point. The proposed algorithm, which is purely linear and thus of very low computational cost and not suffering from initialization problems, is based on the geometric object given by the set of lines incident with the absolute conic. We include experiments which show the good performance of the technique.
\end{abstract}

\section{INTRODUCTION}

The reconstruction of 3D-scenes from images or video sequences taken with uncalibrated cameras is generally performed [3] in three steps starting with the tracking of features in the images, continuing with a projective reconstruction, and finishing with its Euclidean upgrading. This last step involves the obtainment of the intrinsic and extrinsic parameters of the cameras (camera autocalibration). In this paper we deal with this Euclidean upgrading providing a linear algorithm for the autocalibration of ten or more cameras with known pixel shape and varying focal length and principal point, which corresponds to a very frequent practical situation.

Linear algorithms in camera autocalibration are interesting because of their low computational cost, but also because, unlike most algorithms based on the optimization of nonlinear functions [4], they do not need to be initialized with an approximate solution. Thus most applications employ a linear algorithm to obtain a starting solution and then a nonlinear technique to refine it. Linear algorithms for the autocalibration of cameras with zero skew, known principal point and varying focal length are introduced in [5] and [8]. In [1] a linear algorithm is provided which is valid for rotating cameras with known pixel shape. The only linear autocalibration algorithm known by the authors that covers

Work supported by the Ministerio de Ciencia y Tecnologa of the Spanish Government under Project TIC-2001-3069.

\author{
José I. Ronda, Guillermo Gallego
}

\author{
Dpto. de Señales, Sistemas y Radiocomunicaciones \\ Universidad Politcnica de Madrid \\ Ciudad Universitaria, $\mathrm{s} / \mathrm{n}$ \\ E-28040 Madrid, Spain
}

the cases of general camera position and unknown principal point is given in [6], and constitutes a relevant precedent for this work.

The Euclidean upgrading of a projective reconstruction is usually performed by identifying in the projective space the absolute conic lying in the plane at infinity [2][3]. An alternative is to locate an equivalent geometric object as the dual absolute quadric [9], given by the set of planes tangent to the absolute conic, whose estimation is often simpler [3][8]. The present work is motivated by the consideration of another equivalent object consisting in the set of lines intersecting the absolute conic. We call this object the calibration pencil.

The paper is organized as follows. Section 2 summarizes the standard pin-hole camera model and motivates our approach. Section 3 introduces, characterizes and analyzes the calibration pencil. Finally Section 4 applies the theory providing the calibration algorithm and showing experimental results.

\section{MOTIVATION OF CALIBRATION TECHNIQUE}

In this paper we assume that the camera is modeled by the equation $\mathbf{q} \sim P \mathbf{Q}$, (see [3]) where $\sim$ means equality up to a non-zero scale factor, $\mathbf{Q}=(x, y, z, t)^{T}$ denotes the homogeneous coordinates of a spatial point, $\mathbf{q}=(u, v, w)^{T}$ denotes the homogeneous coordinates of an image point, and $P$ is the $3 \times 4$ matrix $P=K(R \mid-R \mathbf{t})$. The intrinsic parameter matrix $K$ is given by

$$
K=\left(\begin{array}{ccc}
\alpha_{u} & -\alpha_{u} \cot \theta & u_{0} \\
0 & \alpha_{v} / \sin \theta & v_{0} \\
0 & 0 & 1
\end{array}\right)
$$

where $u_{0}$ and $v_{0}$ are the affine coordinates of the principal point, $\alpha_{u}$ and $\alpha_{v}$ are the pixel scale factors and $\theta$ is the skew angle between the axes of the pixel coordinates. We denote by $\tau=\alpha_{u} / \alpha_{v}$ the pixel aspect ratio. The matrix $R$ is a rotation matrix which gives the camera orientation, and $\mathbf{t}$ are the coordinates of the camera center. 
We recall here [3] that it is possible to obtain a projective calibration only from image correspondences. This means that, given a set of projected points $\mathbf{q}_{i j}$ obtained with $N$ cameras, $N \geq 2$, we can obtain a set of matrices $\hat{P}_{i}$ and a set of point coordinates $\mathbf{Q}_{j}$ such that $\mathbf{q}_{i j} \sim \hat{P}_{i} \hat{\mathbf{Q}}_{j}$, where $\hat{P}_{i}=P_{i} H^{-1}$ and $\hat{\mathbf{Q}}_{j}=H \mathbf{Q}_{j}$ for some non-singular $4 \times 4$ matrix $H$.

Euclidean calibration can be defined as the obtainment of a matrix $H$ changing the projective coordinates of a given projective calibration to some Euclidean coordinate system, i.e., one in which the absolute conic has equations $x^{2}+y^{2}+$ $z^{2}=t=0$.

If the camera aspect ratio and skew are known, an affine coordinate transformation in the image permits to assume that the internal parameters matrix has the form

$$
K=\left(\begin{array}{ccc}
\alpha & 0 & u_{0} \\
0 & \alpha & v_{0} \\
0 & 0 & 1
\end{array}\right)
$$

We can now introduce the geometric motivation of our method. Observe that the retroprojected lines of image points $(1, \pm i, 0)^{T}$ intersect the absolute conic. In fact, if $\mathbf{Q}=$ $(x, y, z, 0)^{T}$ are the coordinates of the intersection of one of these two lines with the plane at infinity, we have that $(1, \pm i, 0)^{T} \sim P \mathbf{Q}=K R(x, y, z)^{T}$, so $(x, y, z)^{T} \sim$ $R^{T} K^{-1}(1, \pm i, 0)^{T}$, and then $x^{2}+y^{2}+z^{2}=$ $(x, y, z)(x, y, z)^{T}=0$. Therefore, given a projective calibration of such cameras, two lines of the calibration pencil are known for each camera.

If the aspect ratio is unknown but the skew is zero, it is still possible to identify some relevant information in the scene. In fact another straightforward computation shows that the lines obtained by back-projecting points $(1,0,0)$ and $(0,1,0)$ of each camera are orthogonal.

In any of these situations the analysis below will permit to upgrade the projective calibration to an Euclidean calibration with linear algorithms.

\section{CALIBRATION PENCIL}

We first summarize some properties of Plücker coordinates that will be used in the sequel [7]. We consider the set of lines of the projective space $\mathbf{P}^{3}$. Given two different planes of coordinates $\pi=\left(u_{0}, u_{1}, u_{2}, u_{3}\right)$ and $\pi^{\prime}=\left(u_{0}^{\prime}, u_{1}^{\prime}, u_{2}^{\prime}, u_{3}^{\prime}\right)$, we define the Plücker coordinates of the line $\pi \cap \pi^{\prime}$ as the vector of homogeneous coordinates $\mathbf{r}=\left(\pi_{01}, \pi_{12}, \pi_{20}, \pi_{13}\right.$, $\left.\pi_{03}, \pi_{23}\right)^{T}$ given by $\pi_{i j}=u_{i} u_{j}^{\prime}-u_{i}^{\prime} u_{j}$, which can be interpreted as a point of $\mathbf{P}^{5}$.

Let us denote by $\Omega$ the antidiagonal matrix with main antidiagonal $(1,1,1,1,1,1)$. It can be checked that $\mathbf{r}$ verifies $\mathbf{r}^{T} \Omega \mathbf{r}=0$. This relation is homogeneous and quadratic, so it represents a quadric in $\mathbf{P}^{5}$, and it can be checked that any point of this so called Klein quadric corresponds with a line in $\mathbf{P}^{3}$. Two lines of Plücker coordinates $\mathbf{r}$ and $\mathbf{r}^{\prime}$ intersect if and only if they are conjugate with respect to the Klein quadric, i.e., $\mathbf{r}^{T} \Omega \mathbf{r}^{\prime}=0$.

The results which follow will allow us to take advantage of Plücker coordinates to address the problem of Euclidean calibration. Due to lack of space, some proofs of results of the present work are omitted and can be found in [10].

The first important fact is that the set of lines intersecting the absolute conic $C$ is given in Plücker coordinates by the condition $\mathbf{r}^{T} \Sigma \mathbf{r}=0$, where $\Sigma$ is a certain rank-three symmetric $6 \times 6$ matrix defined up to non-zero multiples, which can be interpreted as a quadric in $\mathbf{P}^{5}$.

The first objective is then to recover $\Sigma$. Note that if one knows a set of lines $\left\{\mathbf{r}_{i}\right\}_{i=1, \ldots, N}$ intersecting $C$ and intends to obtain the coefficients of $\Sigma$ solving the homogeneous linear equations $\mathbf{r}_{i}^{T} \Sigma \mathbf{r}_{i}=0$, since these lines also satisfy $\mathbf{r}_{i}^{T} \Omega \mathbf{r}_{i}=0$, what one obtains is a general solution of the form $\Sigma+\lambda \Omega$, which can be interpreted as a pencil of quadrics. $\Sigma$ can nevertheless be obtained since it can be proved that the sum of the elements of the main antidiagonal must be zero independently of the coordinates in $\mathbf{P}^{3}$.

In order to refine initial estimates of a candidate $\Sigma$ obtained by linear methods it is convenient to have a characterization of the space of possible $\Sigma$ matrices.

Result 3.1 A symmetric $6 \times 6$ matrix $\Sigma$ corresponds with a conic in $\mathbf{P}^{3}$ if and only if:

1. $\Sigma \Omega \Sigma=0$,

2. $\Sigma$ is of rank three (the previous condition implies rank $\Sigma \leq$ 3), and

3. The non-zero columns of $\Omega \Sigma$ correspond to Plücker coordinates of lines contained in a plane in $\mathbf{P}^{3}$ (the other possibility, given 1. and 2., is that they are lines through a point).

To check the last condition in the previous result, one can proceed as follows: Let $M$ be the matrix of any three independent columns $\mathbf{r}_{0}, \mathbf{r}_{1}$ and $\mathbf{r}_{2}$ of $\Omega \Sigma$. Let us denote by $M_{i j k}$ the submatrix of $M$ given by the rows $i, j$ and $k$. Then there are two mutually excluding possibilities, either $\operatorname{det} M_{123}=\operatorname{det} M_{246}=\operatorname{det} M_{145}=\operatorname{det} M_{356}=$ 0 , which means that the condition is met or $\operatorname{det} M_{456}=$ $\operatorname{det} M_{135}=\operatorname{det} M_{236}=\operatorname{det} M_{124}=0$, implying that the lines do not define a plane, but a star of lines through a point.

The second important realization is that the absolute conic and consequently the Euclidean structure of space can be retrieved from $\Sigma$. In fact, metric properties can be obtained directly from $\Sigma$, for example two lines are orthogonal if and only if their Plücker coordinates verify that $\mathbf{r}^{T} \sum \mathbf{r}^{\prime}=0$.

In the absence of noise it would be possible to obtain the plane of the absolute conic, i.e., the plane at infinity, as the plane of $\mathbf{P}^{3}$ associated to the plane of $\mathbf{P}^{5}$ given by the kernel of $\Sigma$. However, if $\Sigma$ is estimated from noisy data there is not guarantee that this kernel will correspond to any plane in $\mathbf{P}^{3}$. Let us see how to proceed in this case. Given any 
quadric $\Sigma_{\lambda}=\Sigma+\lambda \Omega$ and a generic line defined by a pair of planes of coordinates $u$ and $v$, we can obtain a symmetric biquadratic expression $S(u, v)$ just by substituting each $\pi_{i j}$ by $u_{i} v_{j}-u_{j} v_{i}$ in the equation of the quadric associated to $\Sigma_{\lambda}$. Since this substitution cancels $\Omega$, the expression only depends on $\Sigma$.

Observe that $S(u, v)=0$ if and only if the planes $u$ and $v$ determine a line intersecting the absolute conic. If $u$ corresponds to the plane at infinity, any other plane $v$ will give, together with $u$, a line in the plane at infinity, which then will intersect the absolute conic. Thus the plane at infinity $u_{\infty}$ is characterized by the property that $S\left(u_{\infty}, v\right)=0$ for all $v$.

Let us see how $\Sigma$ also provides in a straightforward way the projection of the absolute conic on each camera, thus allowing to obtain the camera intrinsic parameters. Observe that an image point belongs to the projected absolute conic if and only if its backprojected line intersects the absolute conic. Computing the Plücker coordinates $\mathbf{r}=\mathbf{r}(u, v, w)$ of the backprojection of image point $(u, v, w)^{T}$ and subtituting in $\mathbf{r}^{T} \Sigma \mathbf{r}=0$ one obtains the desired projected conic.

\section{ALGORITHMS AND EXPERIMENTAL RESULTS}

Now we propose a linear method for finding the plane at infinity and the intrinsic parameters of a set of $N$ cameras $(N \geq 10)$ with known skew and aspect ratio and varying focal length and principal point, based on the preceding analysis. We assume that a projective calibration has already been computed.

1. Use the knowledge of the skew angle and aspect ratio of each camera to change the retinal coordinates so that the intrinsic parameter matrices have the form (2).

2. Back-project the points $(1, \pm i, 0)$ and compute the Plücker coordinates of the corresponding lines $\mathbf{r}_{k}, \overline{\mathbf{r}}_{k}$.

3. Obtain the $6 \times 6$ symmetric matrix $\Sigma$ by solving the linear homogeneous system $\mathbf{r}_{k}^{T} \Sigma \mathbf{r}_{k}=0, \overline{\mathbf{r}}_{k}^{T} \Sigma \overline{\mathbf{r}}_{k}=0$, $k=1, \ldots, N$, together with the condition that the antitrace (sum of the elements of the main antidiagonal) of $\Sigma$ must be zero. Note that $\Sigma$ depends on 21 parameters defined up to scale, so $N=10$ cameras, resulting in $2 N+1=21$ equations, is the minimum number required in order to have an (over)determined system.

4. Compute the biquadratic symmetric expression $S(u, v)$ substituting each $\pi_{i j}$ by $u_{i} v_{j}-u_{j} v_{i}$ in the equation $\mathbf{r}^{T} \Sigma \mathbf{r}=0$ where $\mathbf{r}=\left(\pi_{01}, \pi_{12}, \pi_{20}, \pi_{13}, \pi_{03}, \pi_{23}\right)^{T}$. Calculate the coordinates of the plane at infinity $u_{\infty}$ from the condition that $S\left(u_{\infty}, v\right)=0$ for all $v$, which also leads to a linear homogeneous system of equations.

5. For each camera, compute the Plücker coordinates $\mathbf{r}=\mathbf{r}(u, v, w)$ of the back-projection of a generic image point $(u, v, w)^{T}$ and substitute them in the equation $\mathbf{r}^{T} \sum \mathbf{r}=0$, thus obtaining the projected absolute conic. Obtain from these conic the camera intrinsic parameters.

If the skew is zero it is also possible, as mentioned in Section 2, to identify a couple of intersecting orthogonal lines $\mathbf{r}_{k}, \mathbf{r}_{k}^{\prime}$ for each camera as the back-projections of points $(1,0,0)$ and $(0,1,0)$. With these data it is possible to identify the calibration pencil from equations $\mathbf{r}_{k}^{T} \Sigma \mathbf{r}_{k}^{\prime}=0$. These would require twenty or more cameras and results in a technique related to that of [6]. In both cases it is also possible to improve the linear estimation of $\Sigma$ using its characterization given in result 3.1 .

The previously described algorithms have been tested with synthetic data in a series of experiments involving the reconstruction of a set of 100 points from their projections in 10 to 40 images taken with uncalibrated cameras with varying parameters. The $3 \mathrm{D}$ points lie close to the origin of coordinates of an Euclidean reference and the cameras are located at random positions lying approximately over a sphere centered at the origin and roughly pointing towards it, so that the set of projected points is approximately centered in the virtual CCD. Skew angle and aspect ratio are fixed at respective values $\pi / 2$ and 1 and the other parameters are obtained randomly for each camera and experiment from uniform distributions with support $\left[\alpha_{0}-\Delta \alpha, \alpha_{0}+\Delta \alpha\right]$ for $\alpha,[-A, A]$ for $u_{0}$ and $[-B, B]$ for $v_{0}$ (pixels). The concrete values of the fixed parameters of the experiments have been selected so that the pixel coordinates have values within the range $[-1500,1500]$, however in each image, the points are contained inside a square of side 1500 pixels. After computing the point projections, these are perturbed by the addition of zero-mean Gaussian noise with different variances.

The complete processing for each experiment consists in a projective calibration followed by the computation of the camera parameters by means of the proposed algorithm. Projective calibration is performed in four steps. Firstly, a similarity transformation, $\mathrm{T}=\operatorname{diag}(c, c, 1)$, is applied to all projected points so that a normalization of the coordinates is performed. The value $c$ is the one that makes the average distance from the transformed points to the origin equal to one, in the first image. Secondly, the "Gold Standard" algorithm described in [3] is applied to a pair of images (first and last) to obtain the projective calibration of two cameras. Next, resection is used in the remaining images, so that an initial projective calibration is achieved in the reference frame defined by the two cameras. Finally, a 

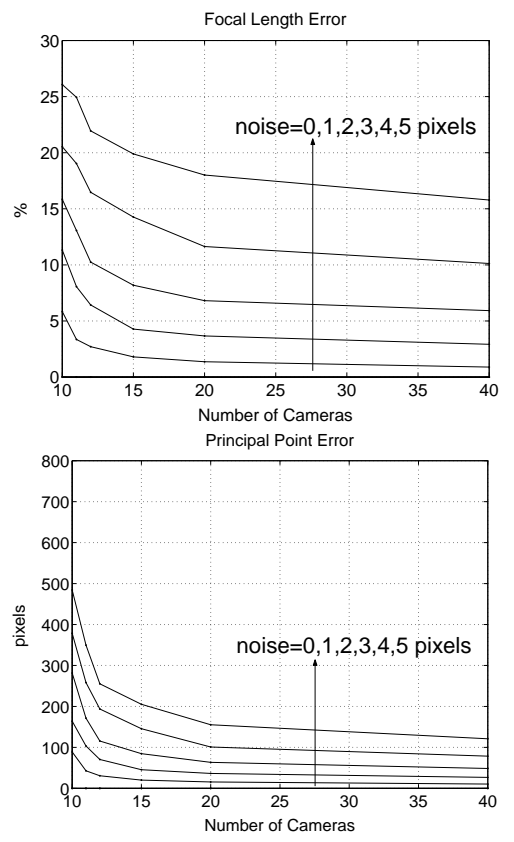

3D Reconstruction Error

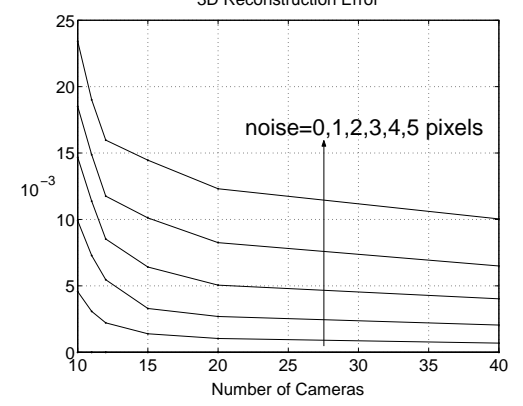

Fig. 1. Top: Average of the relative errors $(\%)$ in the estimation of the focal length as a function of the number of cameras (horizontal axis) and the noise typical deviation, in pixels (increasing curves). Middle: RMS error (pixels) in the estimation of the principal point coordinates. Bottom: RMS of the relative errors in the $3 \mathrm{D}$ reconstruction.

global projective bundle adjustment is performed. In order to measure the error in the intrinsic parameters, the similarity normalization must be inverted.

Figure 1 shows the averaged results for 500 random camera configurations (positions and intrinsic parameters) tested with common parameters: $\alpha_{0}=3.779610^{3}, \Delta \alpha=0.1 \alpha_{0}$, $A=2560 / 4, B=1920 / 4$. For each camera configuration, noise of different levels is added, corresponding to typical deviations $\sigma$ between 0 and 5 pixels, in steps of one. The errors measured are: the relative error in the focal length, the RMS error in the principal point coordinates, $\left(E\left[d^{2}\left(\left(u_{0}, v_{0}\right),\left(\hat{u}_{0}, \hat{v}_{0}\right)\right)\right]\right)^{1 / 2}$, and the error of the corresponding $3 \mathrm{D}$ reconstruction. To measure the latter, the set of reconstructed points is centered, scaled and aligned with the original set of points employing the second order moments of the point distribution. Then the reconstruction error is defined as the RMS of the squared distance between original and reconstructed points divided by the the diagonal of the figure bounding box. The results are competitive in relation with other linear techniques (see, e.g., [1]).

It can be observed from the plots the way the errors decrease if the number of cameras increases. It is worth mentioning that the intrinsic parameters matrices obtained with this algorithm are consistent with the hypotheses on skew and aspect ratio, remaining the average of the relative errors in these two parameters under $0.1 \%$ and $0.15 \%$, respectively.

\section{REFERENCES}

[1] L. Agapito, E. Hayman, I. Reid. Self-Calibration of Rotating and Zooming Cameras. International Journal of Computer Vision 45, pp. 107-127. 2001.

[2] S.J. Maybank and O. Faugeras, A Theory of SelfCalibration of a Moving Camera. International Journal of Computer Vision 8, pp. 123-152. 1992.

[3] R.I. Hartley and A. Zisserman, Multiple View Geometry in Computer Vision. Cambridge University Press, Cambridge, UK, 2000.

[4] A. Heyden and K. Åström. Euclidean reconstruction from image sequences with varying and unknown focal length and principal point. Computer Vision and Pattern Recognition '97, pp. 438-443, 1997.

[5] M. Pollefeys, R. Koch and L. Van Gool. SelfCalibration and Metric Reconstruction in spite of Varying and Unknown Internal Camera Parameters, Proc. ICCV'98.

[6] J. Ponce, On Computing Metric Upgrades of Projective Reconstructions Under the Rectangular Pixel Assumption. Proc. SMILE 2000.

[7] J.G. Semple and G.T. Kneebone, Algebraic Projective Geometry, Oxford University Press, 1998.

[8] Y. Seo, A. Heyden, Auto-calibration from the orthogonality constraints, ICCV'00.

[9] W. Triggs, Auto-calibration and the absolute quadric. In Proc. IEEE Conference on Computer Vision and Pattern Recognition, pages 609-614. 1997.

[10] A. Valdes, J.I. Ronda, G. Gallego, Autocalibration and the calibration pencil, UCM-GTI Internal Report, available at http://www.mat.ucm.es/ avaldes/calibrationpencil.ps 